\title{
Author Correction: Loss of NARS1 impairs progenitor proliferation in cortical brain organoids and leads to microcephaly
}

\author{
Lu Wang, Zhen Li, David Sievert, Desirée E. C. Smith, Marisa I. Mendes, Dillon Y. Chen, Valentina Stanley, \\ Shereen Ghosh, Yulu Wang, Majdi Kara, Ayca Dilruba Aslanger, Rasim O. Rosti, Henry Houlden (1),
}

Gajja S. Salomons \& Joseph G. Gleeson (1)

Correction to: Nature Communications https://doi.org/10.1038/s41467-020-17454-4, published online 12 August 2020.

The original version of this Article omitted a reference to another publication which included overlapping genetic and MRI data for a research participant. This has been added as reference 59 at the end of the Discussion: 'While this paper was under review, a separate paper appeared reporting that mutations in NARS1 associate with neurodevelopmental delay through either biallelic loss or dominant negative effects, impairing NARS1 enzyme activity. This article contained genetic data on family MIC-1433 which overlaps this study ${ }^{59}$.

Accordingly, reference 59 has now been included in the References section as 'Manole, A. et al. De novo and bi-allelic pathogenic variants in NARS1 cause neurodevelopmental delay due to toxic gain-of-function and partial loss-of-function effects. Am. J. Hum. Genet. 107(2), 311-324 (2020).'

In addition, the original version of this Article contained an error in Fig. 1A, where there was an error in the family depiction in generation 1 of pedigree MIC-1433; this has been revised to correctly depict the family. Figure 1D also contained MRI scans that had been previously published in Ref 59; this has been revised to include different and unpublished MRI scans from the same individual. 
The correct version of Fig. 1 is

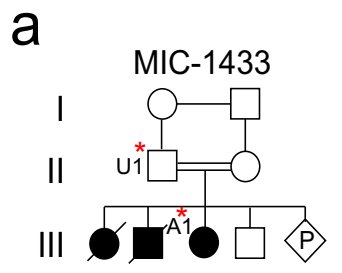

c. $50 \mathrm{C}>\mathrm{T}$, p.T17M

MIC-2116

II

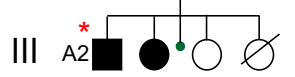

c. [203dupA]; [1067A>C]

p. [M69Dfs*4]; [D356A]

MIC-91

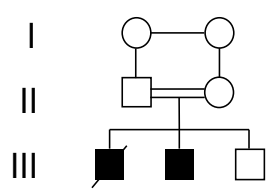

c. $1633 \mathrm{C}>\mathrm{T}$, p.R545C

f NARS1

(asparaginyl-tRNA synthetase, NM_004539.4)
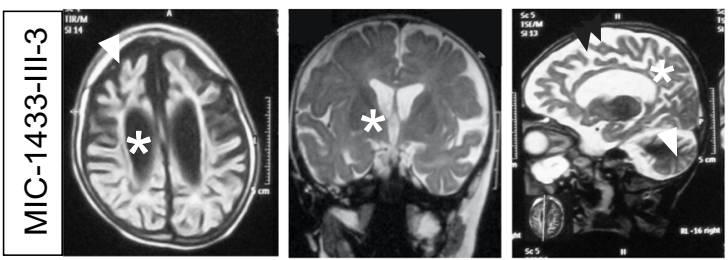

C

HC: $49.2 \mathrm{~cm}$ (-4SD)
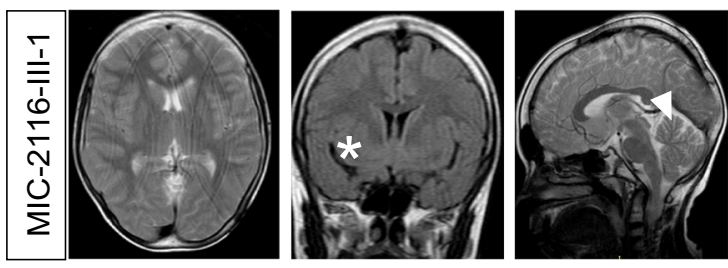

d $\quad \mathrm{HC}: 46.5 \mathrm{~cm}(-4.2 \mathrm{SD})$
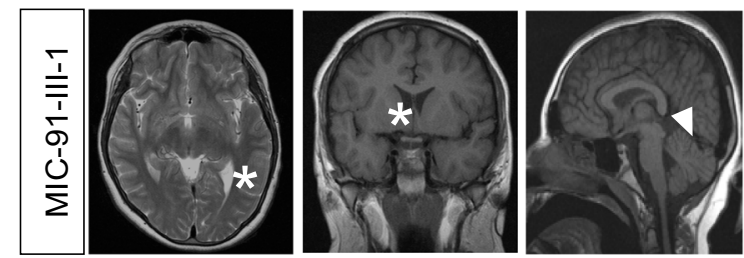

c.50C>T c.203dupA
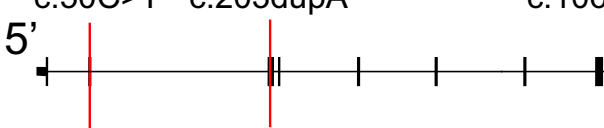

c. $1067 \mathrm{~A}>\mathrm{C}$

Chr18:55267891-55289177

hg19 $5 \mathrm{~kb}$

891-55289177

$>C \quad$ c. $1633 \mathrm{C}>\mathrm{T}$

h
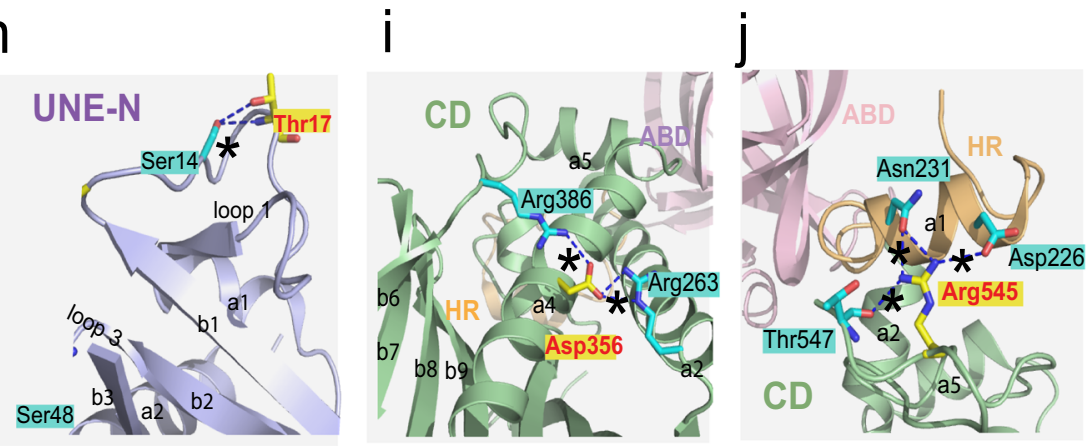

e MIC-1433 (c. 50C>T)

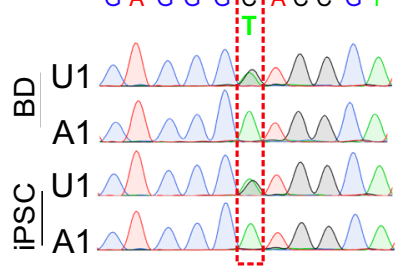

MIC-2116 (c. 1067A>C)

G G T T C

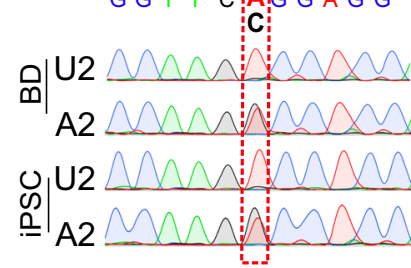

g

NARS1 (NP. 004530.01)

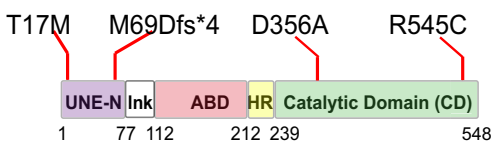

$\mathrm{k}$

H.sapiens H.sapiens (mut)

$P$.troglodytes

M.musculus

G.gallus

$X$.tropicalis

D.rerio

H. sapiens

H.sapiens (mut)

$P$.troglodytes

M.musculus

G.gallus

$X$.tropicalis

$D$.rerio

H. sapiens

H.sapiens (mut)

$P$.troglodytes

M.musculus

G.gallus

$X$.tropicalis

$D$.rerio $\begin{array}{lll}13 & 17 & 21\end{array}$ G S D A T: T D G T' G S D A $M: G D G T$ GSDA T GDG T GNDA T G D G T G S D S T $: G D G T$ G N D S T: $:$ D G T G S D A $: G D G S$ $352 \quad 356 \quad 360$ N R L E D' L V C D N R L E $A: L V C D$ N R LE D L VCD N R LE D L VCD N R LE S L VC D N R LE D L VCD G S D A D : GDG S

$540 \quad 545 \quad 548$ P R F V Q :'R CTP $\begin{array}{ll:l}P R F V & C T P\end{array}$ $P R F \vee Q \quad R: C T P$ P R F V Q R CTP PR F D H $:$ CTP P R F V H R:CTP

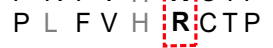


which replaces the previous incorrect version:

a

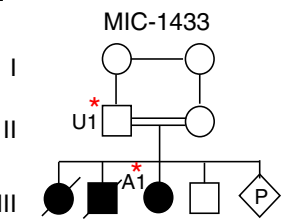

c. $50 \mathrm{C}>\mathrm{T}$, p.T17M

MIC-2116

II<smiles>OC1CC2C[Te]12</smiles>

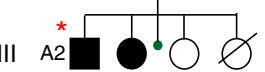

c. [203dupA]; [1067A>C] p. [M69Dfs*4]; [D356A]

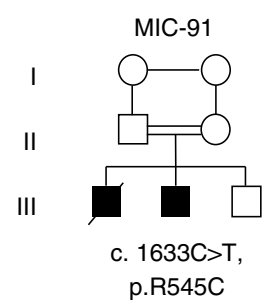

f

NARS1

(asparaginyl-tRNA synthetase, NM_004539.4)
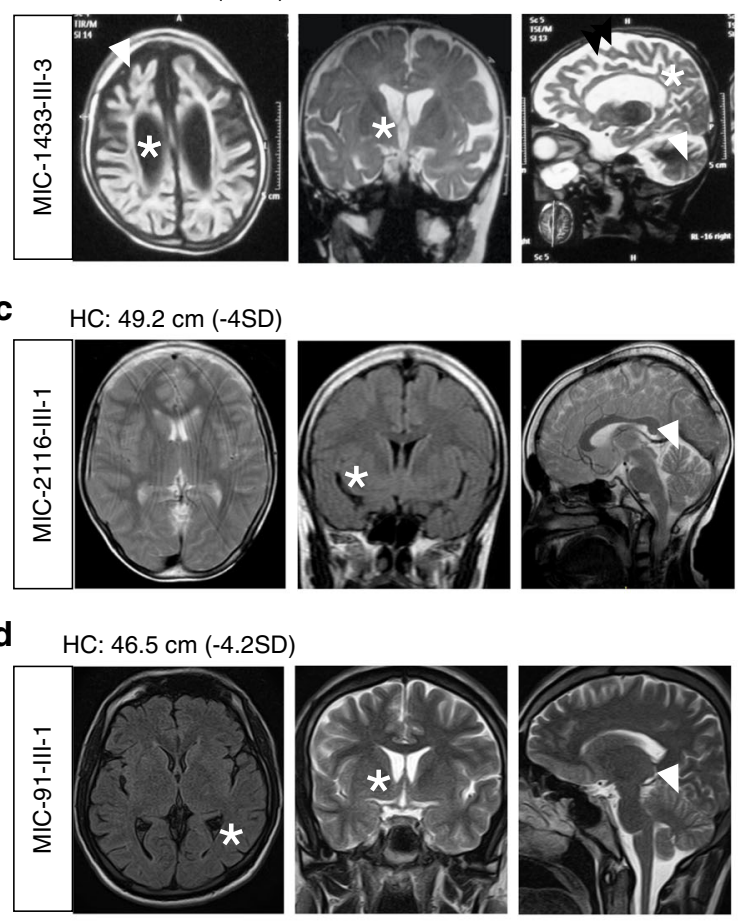

C
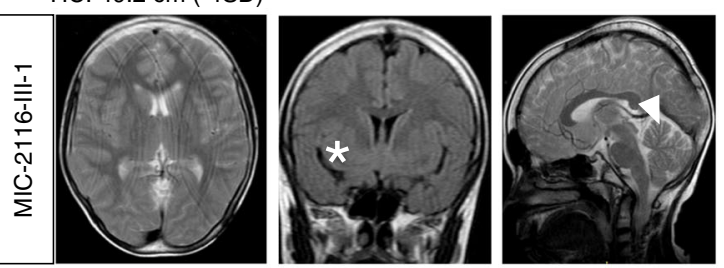

d $\quad \mathrm{HC}: 46.5 \mathrm{~cm}(-4.2 \mathrm{SD})$
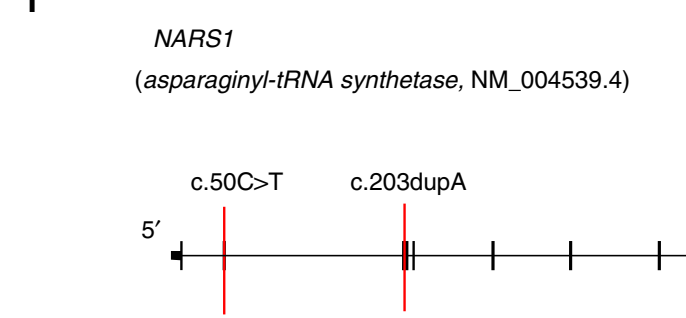

Chr18:55267891-55289177

c. $1067 \mathrm{~A}>\mathrm{C} \quad$ c. $1633 \mathrm{C}>\mathrm{T}$

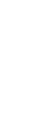

h

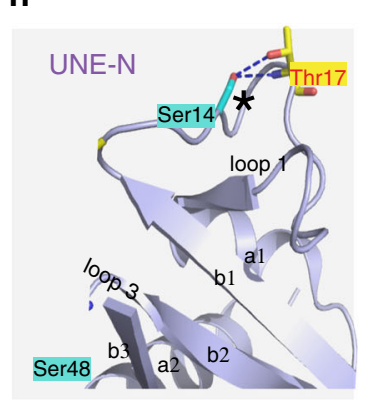

i

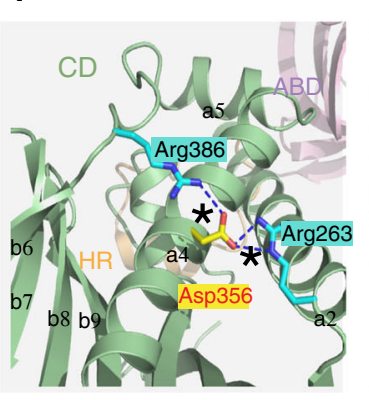

j

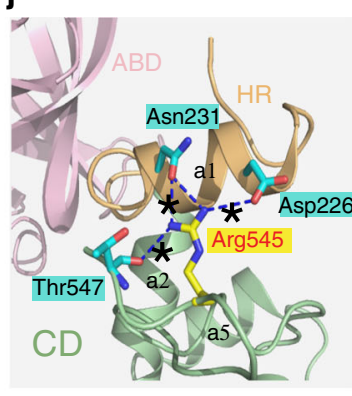

e
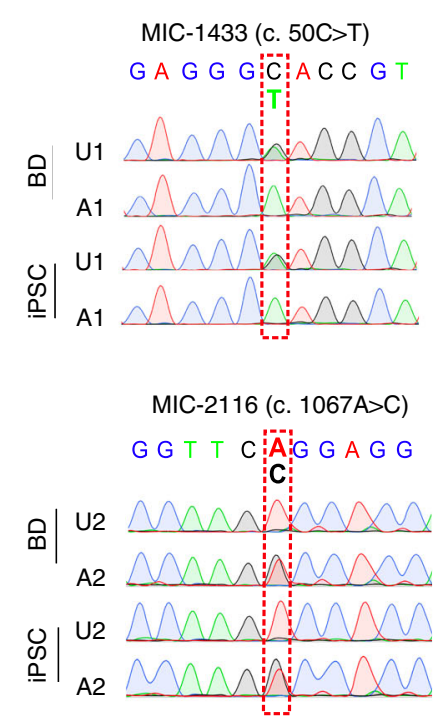

g

NARS1 (NP. 004530.01)

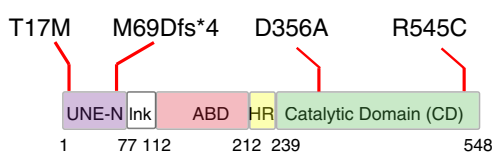

k

H. sapiens

H. sapiens (mut)

$\begin{array}{lll}13 & 17 & 21\end{array}$

P. troglodytes

M. musculus

G. gallus

X. tropicalis

D. rerio

H. sapiens

H. sapiens (mut)

P. troglodytes

M. musculus

G. gallus

X. tropicalis

D. rerio

H. sapiens

H. sapiens (mut)

$P$. troglodytes

M. musculus

G. gallus

$X$. tropicalis

D. rerio $\begin{array}{lll}13 & 17 & 21 \\ 1 & 17 & 1\end{array}$ G S DA $M: G D G$ G S D A T G D G T GNDA $T: G D G T$ G S D S T G D G T G N D S T:G D G T G S DA $D: G D G S$ $352 \quad 356 \quad 360$ N R L E 'D: L VC D NRLE $A: L \vee C D$ NRLE D L VCD NRLE D L LCD N R LE S L V C D $N R L E: D V C D$ G S D A D G G S $540 \quad 545 \quad 548$ P R F V Q $P R F \vee Q: C: C T P$ PRFV Q $R: C T P$ $P R F \vee Q: R$ CTP \begin{tabular}{lll:l:l}
$P$ & $R$ \\
$P$ & $R$ & V D H & $R$ & $C T P$ \\
\hline
\end{tabular} P R F V H R : CTP $P L F V H: R: C T P$

This has been corrected in both the PDF and HTML versions of the Article.

Published online: 15 February 2021 
(c) Open Access This article is licensed under a Creative Commons Attribution 4.0 International License, which permits use, sharing, adaptation, distribution and reproduction in any medium or format, as long as you give appropriate credit to the original author(s) and the source, provide a link to the Creative Commons license, and indicate if changes were made. The images or other third party material in this article are included in the article's Creative Commons license, unless indicated otherwise in a credit line to the material. If material is not included in the article's Creative Commons license and your intended use is not permitted by statutory regulation or exceeds the permitted use, you will need to obtain permission directly from the copyright holder. To view a copy of this license, visit http://creativecommons.org/licenses/by/4.0/.

(C) The Author(s) 2021 